CASE REPORT

\title{
Synchronous ileal and colonic adenocarcinomas associated with Crohn's disease: report of a case with a focus on genetic alterations and carcinogenesis
}

\author{
B Baisse, C Fontolliet, Y S Bian, H Vuilleumier, J Benhattar
}

J Clin Pathol 2004;57:885-887. doi: 10.1136/icp.2003.014811

Patients with Crohn's disease have an increased risk of developing intestinal tumours. However, the carcinogenic mechanisms remain poorly understood. To address this question, this report describes an unusual case of Crohn's disease complicated by synchronous small intestinal and colonic adenocarcinomas. Genetic events in both the tumours and their adjacent mucosae were evaluated and the tumorigenesis of these cancers is discussed.

P atients with Crohn's disease are at increased risk of developing intestinal tumours. ${ }^{1}$ The association of Crohn's disease and adenocarcinoma in the small intestine is uncommon and was first described in 1956. However, this association is not coincidental, and corresponds to a significantly higher relative risk. ${ }^{2}$ Colorectal cancer is the most frequent malignant complication of longstanding ulcerative colitis, although it has not been well studied. Several authors have shown that the risk of developing colorectal cancer in Crohn's disease may be comparable to that in ulcerative colitis. ${ }^{3}$ Unfortunately, the reasons for the increased risk in patients with Crohn's disease remain poorly understood. Indeed, only a few reports have detailed the genetic alterations in Crohn's disease associated with carcinoma.

"Colorectal cancer is the most frequent malignant complication of longstanding ulcerative colitis, although it has not been well studied"

To address this question, we relate an unusual case of Crohn's disease accompanied by synchronous small intestinal and colonic adenocarcinomas with evaluation of the genetic events in both the tumours and their adjacent mucosae.

\section{CASE REPORT}

A diagnosis of ileocolonic Crohn's disease in a 57 year old white woman was established 30 years ago. The patient was treated conservatively and successfully by corticosteroids, and by immunosuppressive and anti-inflammatory drugs. She was admitted with an abscess of the right iliac fossa complicated by a cutaneous fistula. A laparotomy performed five days after antibiotherapy revealed a bulky inflammatory mass including the terminal ileum and the caecum, and a perforation of the right colon. A right hemicolectomy completed by drainage of the fistula was performed. There was no evidence of gross metastatic disease. There was neither vascular invasion nor lymph node involvement. The patient made an excellent postoperative recovery and was fit and well when last reviewed a year after surgery. Pathological examination showed two distinct tumours about $10 \mathrm{~cm}$ apart, namely: (1) a well differentiated adenocarcinoma of the terminal ileum, arising from the areas of chronic transmural inflammation and ulcerations typical of Crohn's disease, extending through the bowel wall and invading the serosa; and (2) a mucinous adenocarcinoma of the right colon expanding from a tubulovillous adenoma.

The normal ileal and colonic mucosae (adjacent and distant from the tumours), in addition to the ileal and colonic carcinomas (two different samples for each tumour), were examined for different molecular alterations. p53 and K-ras mutations and allelic loss at the APC (adenomatous polyposis coli) and DCC (deleted in colon cancer) loci were analysed as described previously using fixed and paraffin wax embedded microdissected tissues. ${ }^{4}$ Microsatellite instability (MSI) was investigated using a panel of microsatellite markers (D2S123, D5S346, D17S250, BAT25, and BAT26), recommended for being the most informative markers for the establishment of MSI in colorectal tumours. ${ }^{5}$ Immunohistochemical analysis was performed using antibodies against $\beta$ catenin (Clone 14; 1/500 dilution; Transduction Laboratories, Lexington, Kentucky, USA), p53 (Clone DO-7; 1/500 dilution; Dako, Glostrup, Denmark), MLH1 (Gl68-15; 1/50 dilution; PharMingen, San Diego, California, USA), and MSH2 (G 219-1129; PharMingen).

\section{RESULTS}

Table 1 summarises the genetic and immunohistochemical analyses. K-ras mutation was detected in the colonic carcinoma and its normal mucosa adjacent to the tumour, but not in the distant mucosa (fig 1). In contrast, no K-ras mutation was detected in the ileal carcinoma and in the corresponding normal mucosa. Mutation of the p53 gene and loss of heterozygosity at the APC and DCC loci were not detected in the colon or the ileum. MSI was not seen in the ileal or the colonic carcinomas, or in the normal mucosae. hMLHI and hMSH2 protein expression was also evaluated and, as expected, expression was normal in the analysed lesions. The immunohistochemical analysis indicated that the cellular distribution of $\beta$ catenin was only membranous, both in normal and tumour tissues.

\section{DISCUSSION}

Patients with inflammatory bowel disease have an increased risk of developing intestinal neoplasia. Many cases of carcinoma of the small bowel complicating Crohn's disease have been reported. ${ }^{6}$ An association of Crohn's disease with adenocarcinoma is predominantly seen in men and occurs most frequently in the distal ileum, in areas of active chronic disease. The prognosis is poor, with a two year survival rate of

Abbreviations: APC, adenomatous polyposis coli; DCC, deleted in colon cancer; MSI, microsatellite instability 


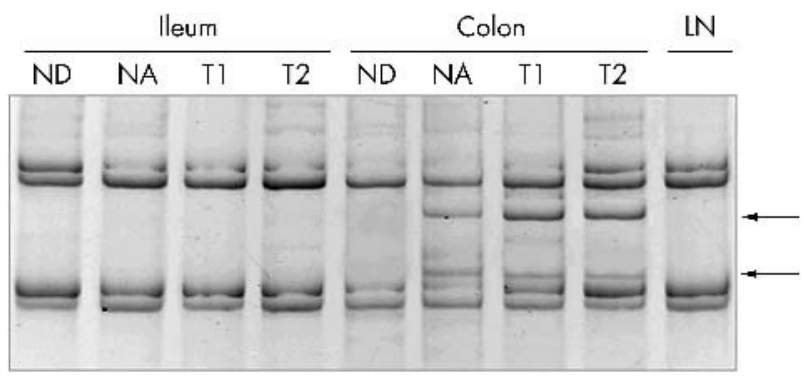

Figure 1 K-ras point mutations by polymerase chain reaction single strand conformation polymorphism analysis. The mobility of normal and tumour tissues was compared with that of normal lymph node tissue (LN). Arrows show mobility shifts corresponding to a GGT $\rightarrow$ GAT transition at codon 12. ND, normal mucosa distant from the tumour; NA, normal mucosa adjacent to the tumour; $\mathrm{T} 1$ and $\mathrm{T} 2$, tumour samples.

only $15-23 \%$. As expected, the small intestinal carcinoma seen in our patient developed in the terminal ileum, in the region of active Crohn's disease. In contrast, our patient was a woman. Despite the advanced stage of the tumour, the prognosis seems to be favourable and no distant dysplasia was observed.

The incidence and risk of colon cancer in patients with Crohn's disease have been debated. Nevertheless, the risk is related to the duration and anatomical extent of the disease. In addition, the relative risk of colon cancer is increased in patients whose Crohn's disease started before the age of 25 years. Colon carcinomas in Crohn's disease have been shown to occur frequently in the right colon, in colonic strictures, and in surgically excluded segments, in addition to anorectal fistulae. The dysplasia to carcinoma sequence for the development of colorectal carcinoma can be applied to the pathogenesis of colon cancer in Crohn's disease. ${ }^{7}$ In our reported case, the colonic carcinoma developed in an area that was not affected by the inflammatory Crohn's disease and was related to a tubulovillous adenoma with low and high grade foci of dysplasia.

\section{"Despite the advanced stage of the tumour, the prognosis seems to be favourable and no distant dysplasia was observed"}

Multicentricity has been noticed in about $20 \%$ of intestinal carcinomas in Crohn's disease, ${ }^{8}$ and the tumours were distributed either in the small intestine or in the colon. To our knowledge, no case with simultaneous ileal and colonic adenocarcinomas has been reported
Take home messages

- We describe an unusual case of Crohn's disease complicated by synchronous small intestine and colonic adenocarcinomas, which were genetically different

- It is probable that the ileal adenocarcinoma, which arose in the area affected by chronic inflammation, was associated with active Crohn's disease, but developed via a K-ras independent pathway

- The colonic carcinoma, which developed in a previous adenoma and was not associated with mismatch repair gene abnormalities, was probably sporadic

The colonic carcinoma in our patient was located in the right colon and had a mucinous differentiation. Although alteration of the mismatch repair genes is generally detected in this group of colonic tumours, MSI was not seen in our patient's tumours.

Primary malignant tumours of the small intestine are rare, accounting for only $1-2 \%$ of all intestinal malignancies, despite a fourfold greater length and a 10 fold greater mucosal area. Several theories have been proposed, including the role of rapid transit time, the presence of detoxifying enzymes that neutralise carcinogens, the high concentration of immunoglobulin A, and the possibility of different molecular mechanisms for the development of carcinomas. The accumulation of K-ras and p53 alterations have been reported during the adenoma/dysplasia-carcinoma sequence in Crohn's associated adenocarcinoma of the small intestine, and in colorectal carcinogenesis. ${ }^{9}$ Nevertheless, a ras independent pathway might also exist in cancers without surrounding adenoma, as demonstrated in the tumour of our patient-the small intestinal carcinoma did not show K-ras mutation.

Our present case represents a very unusual situation, with two synchronous adenocarcinomas, the first located in the ileum and the second in the right colon, discovered in a patient with longstanding ileal Crohn's disease. The molecular analyses showed that the tumours were genetically different. We conclude that there is a high probability that the ileal adenocarcinoma, arising in the area affected by the chronic inflammatory process, was associated with active Crohn's disease. The absence of K-ras mutation in the ileal carcinoma suggests that this tumour developed via a K-ras independent pathway. Conversely, the colonic carcinoma, developing in a previous adenoma and not associated with mismatch repair gene abnormalities, is more likely to be sporadic

Table 1 Genetic alterations in ileal and colonic normal and tumour tissues

\begin{tabular}{lllllllll}
\hline & $\begin{array}{l}\text { K-ras } \\
\text { mutation }\end{array}$ & $\begin{array}{l}\text { p53 } \\
\text { mutation }\end{array}$ & $\begin{array}{l}\text { LOH } \\
\text { DCC }\end{array}$ & $\begin{array}{l}\text { LOH } \\
\text { APC }\end{array}$ & MSI & $\begin{array}{l}\text { IHC MLH1 } \\
\text { and MSH2 }\end{array}$ & $\begin{array}{l}\text { IHC } \\
\text { p53 }\end{array}$ & $\begin{array}{l}\text { IHC } \beta \\
\text { catenin }\end{array}$ \\
\hline Ileum ND & wt & wt & No & No & - & + & - & - \\
Ileum NA & wt & wt & No & No & - & + & - & - \\
lleum T1 & wt & wt & No & No & - & + & - & - \\
lleum T2 & wt & wt & No & No & - & + & - & - \\
Colon ND & wt & wt & No & No & - & + & - & - \\
Colon NA & GAT & wt & No & No & - & + & - & - \\
Colon T1 & GAT & wt & No & No & - & + & - & - \\
Colon T2 & GAT & wt & No & No & - & + & - & - \\
LN & wt & wt & No & No & - & + & - & -
\end{tabular}

APC, adenomatous polyposis coli; DCC, deleted in colon cancer; GAT, mutation GGT $\rightarrow$ GAT at codon 12; $I H C$ immunohistochemistry; LN, normal lymph node (as control material); LOH, loss of heterozygosity; MSI,

microsatellite instability; NA, normal mucosa adjacent to the tumour; ND, normal mucosa distant from the tumour; $\mathrm{T1}$, tumour (first sample); T2, tumour (second sample); wt, wild type; -, negative. 


\section{Authors' affiliations}

B Baisse, C Fontolliet, Y S Bian, J Benhattar, Institute of Pathology, Centre Hospitalier Universitaire Vaudois, CH-1011 Lausanne,

Switzerland

H Vuilleumier, Surgical Department, Centre Hospitalier Universitaire Vaudois

Correspondence to: Dr J Benhattar, Institute of Pathology, Centre Hospitalier Universitaire Vaudois, Bugnon 25, CH-1011 Lausanne, Switzerland; jean.benhattar@chuv.hospvd.ch

Accepted for publication 15 March 2004

\section{REFERENCES}

1 Lewis JD, Deren JJ, Lichtenstein GR. Cancer risk in patients with inflammatory bowel disease. Gastroenterol Clin North Am, 1999;28:459-77.
2 Ribeiro MB Greenstein AJ Yamazaki Y Adenocarcinoma of the small intestine in Crohn's disease. Gynecol Obstet 1991;173:343-9.

3 Choi PM, Zelig MP. Similarity of colorectal cancer in Crohn's disease and ulcerative colitis: implications for carcinogenesis and prevention. Gut 1994;35:950-4.

4 Baisse B, Bouzourene $\mathrm{H}$, Saraga EP, et al. Intratumor genetic heterogeneity in advanced human colorectal adenocarcinoma. Int J Cancer 2001;93:346-52.

5 Boland CR, Thibodeau SN, Hamilton SR, et al. National Cancer Institute workshop on microsatellite instability for cancer detection and familial predisposition: development of international criteria for the determination of microsatellite instability in colorectal cancer. Cancer Res 1998:58:5248-57.

6 Savoca PE, Ballantyne GH, Cahow C. Gastrointestinal malignancies in Crohn's disease. Dis Colon Rectum 1990;33:7-11.

7 Sigel JE, Petras RE, Lashner BA, et al. Intestinal adenocarcinoma in Crohn's disease. Am J Surg Pathol 1999;23:651-5.

8 Michelassi F, Testa G, Pomidor WJ, et al. Adenocarcinoma complicating Crohn's disease. Dis Colon Rectum 1993;3:654-61.

9 Rashid A, Hamilton SR. Genetic alterations in sporadic and Crohn'sassociated adenocarcinomas of the small intestine. Gastroenterology 1997;113:127-5. 